\title{
The dynamic expression of aquaporins 1 and 4 in rats with hydrocephalus induced by subarachnoid haemorrhage
}

\author{
Chun-Yan Long ${ }^{1,2}$, Gui-Qin Huang ${ }^{3}$, Qiong $\mathrm{Du}^{1}$, Li-Qing Zhou ${ }^{4}$, Jing-Hua Zhou ${ }^{1,2}$ \\ ${ }^{1}$ Department of Neurology, The First College of Clinic Medical Sciences, China Three Gorges University, Yichang 443003, Hubei \\ Province, China, ${ }^{2}$ Institute of Neurology, China Three Gorges University, Yichang 443003, Hubei Province, China, ${ }^{3}$ Experimental \\ Animal Center, China Three Gorges University, Yichang 443002, Hubei Province, China, ${ }^{4}$ Zigui Maternal and Child Health Care \\ Hospital, Yichang 443003, Hubei Province, China
}

\begin{abstract}
Introduction: Hydrocephalus is a common complication of subarachnoid haemorrhage (SAH). As transmembrane water channels, aquaporins 1 and 4 (AQP1 and AQP4) are involved in the pathogenesis of hydrocephalus. We aimed to assess the association between the expressions of AQP1 and AQP4 and the severity and duration of hydrocephalus after SAH.

Material and methods: A double haemorrhage model by injection of autologous blood into the cisterna magna was used to induce SAH in rats. Sham rats received the same procedures, but with the injection of normal saline. The SAH group was divided into the SAH with hydrocephalus group and SAH without hydrocephalus group after identifying hydrocephalus histologically. $A Q P 1$ and $A Q P 4$ in ventricle regions were detected by immunofluorescence, quantitative polymerase chain reaction ( $q P C R)$ and western blot.

Results: Hydrocephalus was the most severe at day 3 after SAH. AQP1 and AQP4 MRNA and protein levels increased at day 1 and peaked at day 3. The SAH with hydrocephalus group had a higher expression of AQP1 and AQP4 than the SAH without hydrocephalus group. Higher AQP1 levels were found at the apical and basolateral membrane of the choroid plexus epithelium, while higher AQP4 levels were found in the ependymal cells. A positive correlation between the relative lateral ventricle area and the ratio of $A Q P 1 / A Q P 4$ proteins was identified.

Conclusions: $A Q P 1$ and $A Q P 4$ are remarkably correlated with the severity of hydrocephalus induced by $S A H . A Q P 1$ and AQP4 are potential drug targets for developing therapeutic strategies against hydrocephalus.
\end{abstract}

Key words: subarachnoid haemorrhage, hydrocephalus, aquaporin-1, aquaporin-4.

\section{Introduction}

Hydrocephalus is a common complication after subarachnoid haemorrhage $(\mathrm{SAH})$, which is characterized by dilated ventricle and increased intracranial pressure $[18,25]$. The incidence of hydrocephalus in SAH patients is as high as 6-67\%, depending on specific background and clinical circumstances [1,12], but most recent studies report an overall $20-30 \%$ incidence of hydrocephalus after SAH $[9,17]$. The onset of hydrocephalus can be acute (within $48 \mathrm{~h}$ after SAH) or, more rarely, chronic (weeks and even months after the haemorrhage) $[22,46,48]$. Regardless of its timing, hydrocephalus significantly impairs the patient's central nervous system and leads to functional deterio-

\section{Communicating author}

Jinghua Zhou, MD, Department of Neurology, The First College of Clinic Medical Sciences, China Three Gorges University, 443003 , Yichang, China, phone: +86-15971663190, e-mail: Ifydhgz@163.com 
ration, especially with intraventricular haemorrhage [9]. Since patients with hydrocephalus after SAH have high rates of disability and mortality $[18,25]$, elucidating the molecular mechanisms underlying hydrocephalus would provide a theoretical basis that is urgently needed to seek better therapeutic approaches.

Aquaporins (AQPs) are a group of bidirectional transmembrane water channels that are involved in maintaining brain water homeostasis $[4,6]$. Their discovery has greatly improved our knowledge on how water is absorbed and released across various membranes. At least seven AQP subtypes have been identified in the rodent brain, and they are involved in several diseases featured by cerebral oedema, including hydrocephalus $[2,37,45]$. AQPs in the central nervous system facilitate water transport among the major brain compartments: the cerebrospinal fluid (CSF) space (including cerebral ventricles and subarachnoid space), the brain parenchyma (intracellular and extracellular space) and the intraventricular compartment $[37,44,45,49]$. Hydrocephalus occurs due to an abnormal accumulation of CSF, which is usually resulting from the blockage of CSF outflow in the ventricles or in the subarachnoid space over the brain [45]. Two AQPs, AQP1 and AQP4, have been reported to play key roles in several hydrocephalus models and are thus potential drug targets $[36,45]$.

AQP1 and AQP4 are involved in the generation of CSF and the removal of excess water from the brain, respectively [36,37]. The up-regulation of AQP4 in animal hydrocephalus models has been reported, which suggested that this might be due to a compensatory effect of hydrocephalus $[14,36]$. AQP1 is involved in CSF production, and its change in hydrocephalus is controversial. Mao et al. reported that the expression of AQP1 was unchanged in kaolin-induced hydrocephalus [31], while another report has indicated that its expression was reduced in non-obstructive hydrocephalus induced by choroid plexus hyperplasia [41]. Besides, AQP1 levels were reported to be elevated in communicative hydrocephalus related to choroid plexus papilloma [29]. Furthermore, Wang et al. reported that the ventricular size was reduced in AQP1-deficient mice following kaolin-induced hydrocephalus, suggesting a potential correlation between AQP1 expression and ventricular size [47]. This also implies that the down-regulation of AQP1 might be beneficial to the treatment of hydrocephalus. Nevertheless, to the best of our knowledge, there are very few studies on the expression of AQP1 and AQP4 in hydrocephalus after SAH. The dynamic changes of AQP1 and AQP4 in SAH complicated with hydrocephalus still remain largely unknown.

In the present study, we established a rat model of hydrocephalus after $\mathrm{SAH}$, and investigated the dynamic changes of AQP1 and AQP4 expressions, at the mRNA and protein levels. The subcellular location of AQP1 and AQP4 in the ventricle region of rats with and without hydrocephalus after SAH was also explored. In addition, the correlations between AQP1/4 expression in the peripheral region of the lateral ventricle and the size of the lateral ventricle area were further investigated. Our delineation on the expression patterns of AQP1 and AQP4 during the course of hydrocephalus after $\mathrm{SAH}$ could assist the development and implementation of AQP1/4-targeted drugs for the nonsurgical therapy of this disease.

\section{Material and methods \\ Animals}

Adult male Sprague-Dawley rats (230-250 g, $n=120)$ were purchased from the Experimental Animal Centre of China Three Gorges University. Rats were housed with free access to food and water in a room maintained at $25^{\circ} \mathrm{C}$ and a $12 / 12 \mathrm{~h}$ light/dark cycle. The rats were randomly assigned into two groups: sham group $(n=24)$ and SAH group $(n=96)$. According to the presence or absence of hydrocephalus after SAH, the rats in the SAH group were further randomized to the $\mathrm{SAH}$ with hydrocephalus $(\mathrm{SAH} / \mathrm{HyC})$ group and $\mathrm{SAH}$ without hydrocephalus (SAH w/t HyC) group. The animal study was approved by the Animal Care and Use Committee of China Three Gorges University (CTGU), and all experiments involving animals were performed according to the policies of the Chinese animal research committees and guidelines of the US National Institute of Health (NIH Publication No. 96-23, revised 1996).

\section{The SAH model}

A modified rat double haemorrhage model was used to induce $\mathrm{SAH}$, as previously reported $[8,13]$. The rats were anesthetized intraperitoneally using $10 \%$ chloral hydrate $(350 \mathrm{mg} / \mathrm{kg})$. The head was fixed in a stereotactic frame (Stoelting, USA) in the prone position. After a vertical midline incision in the sub-occipital region was made, the atlanto-occipital membrane was exposed by dividing the nuchal muscle layers. A 25-gauge needle was inserted into the cisterna magna, and 0.06-0.1 ml of CSF was slow- 
ly withdrawn. Non-heparinized autologous blood (0.1 $\mathrm{ml} / 100 \mathrm{~g}$ body weight) from the caudal artery was injected into the cisterna magna over 2 min to induce the first $\mathrm{SAH}$. The rats were kept prone with $30^{\circ}$ head down position for $30 \mathrm{~min}$ to ensure blood distribution around the basal arteries. The same operation was conducted $48 \mathrm{~h}$ after the first induction of $\mathrm{SAH}$. The rats in the sham group were subjected to the same experimental procedures except that normal saline but not autologous blood was injected. Successful induction of SAH was verified by the presence of clotted blood in the cisterna magna and the basilar cistern at $14 \mathrm{~h}$ after the first SAH operation (Fig. 1).

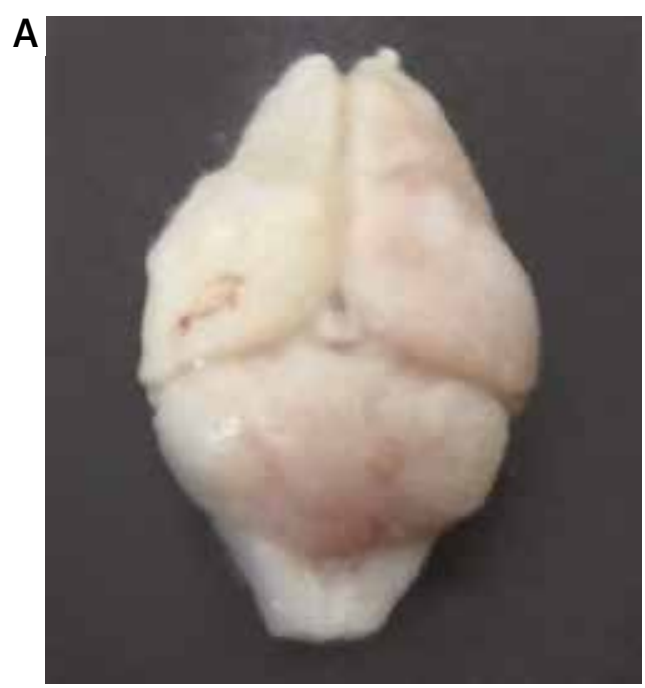

B

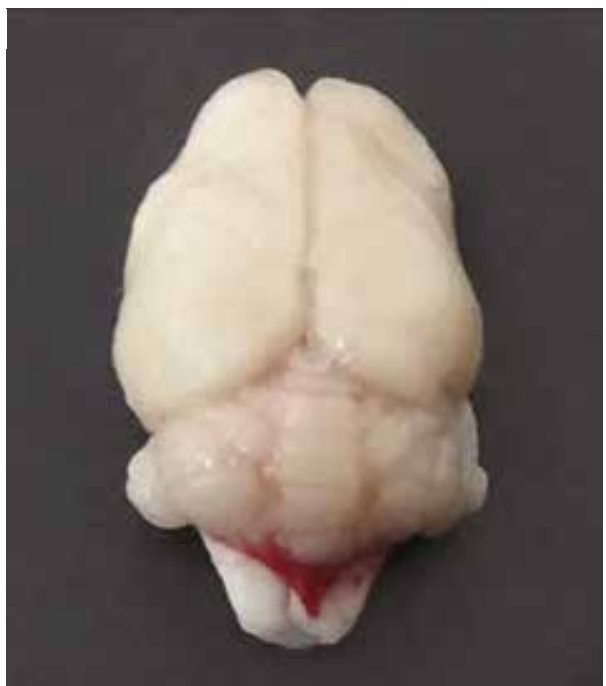

\section{Neurological scoring}

A modified scoring method based on the report of Sugawara et al. [42] was used to assess the neurological status of the rats on days $1,3,7$, and 14 after SAH induction. The scores were determined in a blinded fashion by two different investigators to avoid bias. An 18-point scoring system was used to evaluate the deficits of sensation and movement (Table I). The maximal obtainable score is 18 , and high score means better function.

\section{Tissue collection and preparation}

After anaesthesia with $10 \%$ chloral hydrate (350 $\mathrm{mg} / \mathrm{kg}$ ) intraperitoneally, the rats were per-
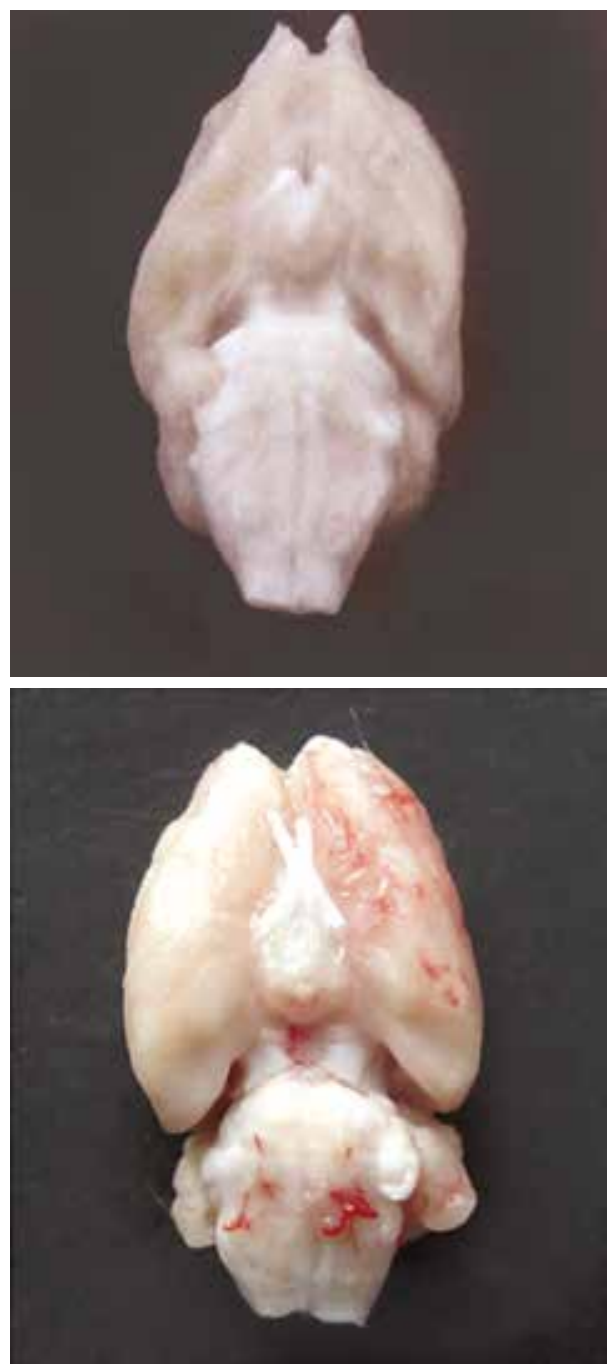

Fig. 1. Verification of the successful establishment of the experimental SAH model in rats. Representative images show the clotted blood in the cisterna magna and the basilar cistern of brains $14 \mathrm{~h}$ after the induction of SAH. A) The rat brain in the sham group. B) The rat brain in the $\mathrm{SAH}$ group. Scale bar, $5 \mathrm{~mm}$. 
Table I. The scoring standard for neurological function evaluation of rats after $\mathrm{SAH}$. The table shows the neurological scoring adopted to evaluate the neurological status of animals at day 1, 3, 7, and 14 post-SAH. The maximal score is 18

\begin{tabular}{|lcccc|}
\hline Test & \multicolumn{3}{c|}{ Score } & \\
\cline { 2 - 4 } & 0 & 1 & 2 & 3 \\
\hline $\begin{array}{l}\text { Spontaneous activity } \\
\text { (in cage for 5 min) }\end{array}$ & No movement & $\begin{array}{c}\text { Barely moves } \\
\text { position }\end{array}$ & $\begin{array}{c}\text { Moves but does not } \\
\text { approach at least three sides } \\
\text { of the cage }\end{array}$ & $\begin{array}{c}\text { Moves and approaches at } \\
\text { least three sides of the cage }\end{array}$ \\
\hline $\begin{array}{l}\text { Spontaneous movements } \\
\text { of all limbs }\end{array}$ & No movement & $\begin{array}{c}\text { Slight movement } \\
\text { of limbs }\end{array}$ & $\begin{array}{c}\text { Moves all limbs but } \\
\text { slowly }\end{array}$ & $\begin{array}{c}\text { Move all limbs same as } \\
\text { pre-SAH }\end{array}$ \\
\hline $\begin{array}{l}\text { Movements of forelimbs } \\
\text { (outstretching while held } \\
\text { by tail) }\end{array}$ & No movement & $\begin{array}{c}\text { Slight outreaching } \\
\text { Climbing wall of the wire cage }\end{array}$ & $\begin{array}{c}\text { Outreach is limited and } \\
\text { less than pre-SAH }\end{array}$ & Outreach same as pre- \\
\hline $\begin{array}{l}\text { Reaction to touch on both } \\
\text { sides of the trunk }\end{array}$ & Fails to climb & No response & Weak response & Normal climbing \\
\hline Reaction to vibrissae touch & No response & Weak response & Normal response \\
\hline
\end{tabular}

fused sequentially with $0.9 \%$ normal saline solution $\left(4^{\circ} \mathrm{C}\right)$ and $4 \%$ buffered paraformaldehyde $\left(4^{\circ} \mathrm{C}\right)$. The brains were collected and immersed in $4 \%$ buffered paraformaldehyde $\left(4^{\circ} \mathrm{C}\right)$ for $24 \mathrm{~h}$, and then made into $10 \mu \mathrm{m}$ coronal cryosections of three levels (preoptic area, striatum, and hippocampus) after gradient dehydration using sucrose and embedding. Hydrocephalus was identified histologically after hematoxylin and eosin (HE) staining, and the coronal cryosections at the level of the striatum were chosen for immunofluorescence analysis.

Alternatively, brains were removed and divided into left and right hemispheres on ice. The left hemispheres were fixed with $4 \%$ buffered paraformaldehyde $\left(4^{\circ} \mathrm{C}\right)$ for $24 \mathrm{~h}$, and made into frozen sections of three levels, as above, which were used to identify hydrocephalus. The right hemispheres corresponding to the lateral ventricle were divided into the anterior and posterior regions. The two regional samples were harvested on ice and stored at $-80^{\circ} \mathrm{C}$ for reverse transcription-quantitative polymerase chain reaction (RT-qPCR) and western blot.

\section{HE staining and hydrocephalus identification}

HE staining was performed according to standard procedures, and HE-stained coronal sections of the above-mentioned three levels were photographed under a light microscope. Histomorphology analysis was conducted by a computer-assisted tool (Photoshop CS5, Adobe, San Jose, CA, USA) to detect the lateral ventricle and the total brain area. The relative lateral ventricle area was calculated as the ratio of lateral ventricle area/total brain area, and the average of the three slices was considered as the value for each rat, as previously reported [19]. Hydrocephalus was defined as relative lateral ventricle area $>+3$ SDs (standard deviations) above the mean value of the sham rats, as previously described [33].

\section{Immunofluorescence}

The cryosections mentioned above were washed with PBS (3 min/time, five times), and blocked in $10 \%$ normal goat serum (Biosynthesis Biotechnology, Beijing, China) for $1 \mathrm{~h}$. Without washing, sections were incubated with rabbit anti-AQP-1 polyclonal antibody (1 : 500 dilution, Abcam, USA), or mouse anti-AQP-4 monoclonal antibody (1 : 100 dilution, Abcam, USA) overnight at $4^{\circ} \mathrm{C}$. After washing with PBS (3 min/time, five times), the sections were incubated with rhodamine-conjugated goat anti-rabbit IgG (1 : 100 dilution, Kerui Biotechnology, Wuhan, China) or FITC conjugated goat anti-mouse IgG ( 1 : 100 dilution, Kerui Biotechnology, Wuhan, China) secondary antibody at $37^{\circ} \mathrm{C}$ for $1 \mathrm{~h}$. After washing and mounting, the slides were visualized using an inverted fluorescence microscope (Leica, Germany).

\section{RT-qPCR}

Total RNA was extracted from tissues corresponding to the region of lateral ventricle and the region surrounding lateral ventricle using a RNA simple Total RNA Kit (Tiangen Biotech, Beijing, China). 
The concentration and purity of total RNA was detected and verified by ultraviolet spectrophotometry. Reverse transcription was conducted using a RevertAid First Strand cDNA Synthesis Kit (Thermo Scientific, China). Quantitative PCR (qPCR) was performed using SYBR ${ }^{\circledR}$ Premix Ex Taq ${ }^{\mathrm{TM}}$ II (TaKaRa Bio Inc, China) and the Mx3005P Real-Time PCR System (Agilent Stratagene, Germany). The primers of AQP1, AQP4, and GAPDH have been reported previously [34,51]. The sequences are: AQP1, 5'-CCG AGA CTT AGG TGG CTC AG-3' (forward) and 5'-TCA TGC GGT CTG TAA AGT CG-3' (reverse); AQP4, 5'-GAA GAC AGC ACC TGT GAT AG-3' (forward) and 5'-CGC TGA GCA GAA CAA AGA TG (reverse); GAPDH, 5'-AGG TCG GTG TGA ACG GAT TTG-3' (forward) and 5'-GTA GAC CAT GTA GTT GAG GTC-3' (reverse). qPCR for each sample was performed in triplicate. GAPDH was used as the internal control, and the fold-changes of AQP1 and AQP4 were calculated according to the $2^{-\Delta \Delta \mathrm{Ct}}$ method [28].

\section{Western blot}

Tissues corresponding to the region of the lateral ventricle and the region surrounding the lateral ventricle were separated from the frozen brain samples, and homogenized in radioimmunoprecipitation assay (RIPA) buffer (Beyotime Biotechnology, China) with the protease inhibitor, phenylmethylsulfonyl fluoride (PMSF) (Beyotime Biotechnology, China). After incubation for 30 min on ice, the homogenate was centrifuged at $12,000 \mathrm{rpm}$ for $5 \mathrm{~min}$ at $4^{\circ} \mathrm{C}$. The supernatant was obtained and the protein concentration was estimated using a BCA-kit (Beyotime Biotechnology, China). The samples (40 $\mu \mathrm{g}$ per lane) were separated by $12 \%$ sodium dodecyl sulfate polyacrylamide gel electrophoresis (SDS-PAGE) and electro-transferred onto PVDF membranes (Millipore, Bedford, MA, USA). The membranes were blocked with $5 \%$ skimmed milk dissolved in TBST (TBS with $0.1 \%$ Tween 20) for $2 \mathrm{~h}$ at room temperature. The membranes were incubated with three different primary antibodies overnight at $4^{\circ} \mathrm{C}$ : polyclonal rabbit anti-AQP1 (1 : 300, Boster Biotech, Wuhan, China), polyclonal rabbit anti-AQP4 (1 : 2000, Proteintech, Wuhan, China), and polyclonal rabbit anti-GAPDH (1 : 1000, Xianzhi Biotechnology, Hangzhou, China) antibodies. GAPDH was used as a loading control. After washing with PBST (PBS with $0.1 \%$ Tween 20, $5 \mathrm{~min} /$ time, six times), the membranes were incu- bated with the HRP-conjugated goat anti-rabbit IgG (1 : 50 000, Boster Biotech, Wuhan, China) secondary antibody for $2 \mathrm{~h}$ at $37^{\circ} \mathrm{C}$. The bands were visualized by enhanced chemiluminescence (ECL) reagents (Thermo, China) and were exposed to X-ray films. Optical densities were acquired by the Glyko Bandscan software (Glyko, Novato, CA, USA). The optical density of targeted proteins was normalized with respect to that of GAPDH. All experiments were repeated at least three times.

\section{Statistical analysis}

Data were analysed using SPSS 18.0 and GraphPad Prism 5.0, and expressed as means \pm SEM (the standard error of the mean). The independent sample $t$ test was used to assess the differences between two groups. The statistical differences among groups were analysed by one-way analysis of variance (ANOVA) followed by the Student-Newman-Keuls (SNK) test. Correlations between relative lateral ventricle area and the expression of AQP1 and AQP4 protein or the ratio of AQP1 and AQP4 protein expression were calculated using the Pearson test. The 95\% upper limit of reference value for the ratio of AQP1 and AQP4 protein expression in $\mathrm{SAH} w / \mathrm{t} \mathrm{HyC} \mathrm{group} \mathrm{was} \mathrm{assessed} \mathrm{using} \mathrm{mean} \pm 1.64$ SDs. Statistical significance was assumed if $p<0.05$.

\section{Results \\ Mortality and neurobehavioral evaluation}

As previously described $[8,47]$, the haemorrhage model often results in accidental rat death. In this study, all rats in the sham group survived during the observation period, while 12 rats in the SAH group died after the operation, with a total mortality rate of $10.00 \%$. The mortality for the $\mathrm{SAH}$ group was $16.67 \%$ (4 of 24) at day 1, $12.50 \%$ (3 of 24) at day 3, $12.50 \%$ (3 of 24) at day 7, and $8.33 \%$ (2 of 24) at day 14 . The survivors were used for the subsequent experiments. As shown in Table II, neurological scores decreased in most rats after SAH. These rats were characterized by drowsiness, anorexia, decreased limb movement, and decreased activity. Compared with the sham group, the rats in the SAH group had most significantly decreased neurological scores at days 1 and 3, and relative decreased scores at days 7 and 14 (Table II, $p<0.001$ ). The rats in the sham group had no noticeable deficit. Although 
Table II. Evaluation of neurologic dysfunctions in rats after SAH. Compared with the sham group, ${ }^{* * *} p<0.001$. For the sham group, $n=\mathrm{X}$ at $\mathrm{d} 1, n=\mathrm{X}$ at $\mathrm{d} 3, n=\mathrm{X}$ at $\mathrm{d} 7$, and $n=\mathrm{X}$ at $\mathrm{d} 14$; for the SAH group, $n=\mathrm{X}$ at d1, $n=\mathrm{X}$ at d3, $n=\mathrm{X}$ at d7, and $n=\mathrm{X}$ at d14

\begin{tabular}{|lcccc|}
\hline Groups & D1 & D3 & D7 & D14 \\
\hline Sham group & $16.17 \pm 0.48$ & $17.17 \pm 0.31$ & $18.00 \pm 0.00$ & $18.00 \pm 0.00$ \\
\hline SAH group & $9.95 \pm 0.65^{\star \star \star}$ & $11.48 \pm 0.52^{\star \star \star}$ & $15.62 \pm 0.37^{\star \star \star}$ & $16.68 \pm 0.24^{\star \star \star}$ \\
\hline
\end{tabular}

the neurological status of SAH rats were improved gradually over time, significantly low of neurological scores could be observed at days 1,3 , and 7 in the SAH w/t HyC group (Fig. 2, $p<0.05$ ), and at all time points in the SAH/HyC group (Fig. 2, $p<0.001$ ). Compared with the SAH w/t HyC group, neurological scores in the SAH/HyC group were significantly lower at all time points (Fig. 2, $p<0.01$ ).

\section{Relative lateral ventricle area and hydrocephalus}

Hydrocephalus after SAH was assessed by $\mathrm{HE}$ staining of the preoptic area, the striatum, and the hippocampus of rat brains (Fig. 3A, B). Thirty-six rats had hydrocephalus, while 48 rats did not reach the criteria for hydrocephalus, resulting in an occurrence of hydrocephalus of $42.9 \%$ (36/84). As shown in Figure 3C, compared with the sham group, the relative lateral ventricle area was significantly higher in the SAH w/t HyC group at days 1 and 3 $(p<0.05)$, and increased more significantly in the $\mathrm{SAH} / \mathrm{HyC}$ group at all time points $(p<0.001)$. Notably, the increase was the most obvious at day 3 in the SAH/HyC group (8.68 $\pm 0.46 \%$ vs. $3.27 \pm 0.22 \%$, $p<0.001)$. In line with the attenuation of the neurological status, the relative lateral ventricle area in the $\mathrm{SAH} / \mathrm{HyC}$ group was gradually decreased (6.93 $\pm 0.24 \%$ at day 7 , and $5.91 \pm 0.21 \%$ at day 14 ). Even at day 14 , the relative lateral ventricle area in the $\mathrm{SAH} / \mathrm{HyC}$ group was still larger than that at day 1 ( $5.91 \pm 0.21 \%$ vs. $5.18 \pm 0.08 \%)$.

\section{Localization and expression of AQP1 and AQP4 in the ventricle region by immunofluorescence}

Immunofluorescence was used to detect the localization and expression of AQP1 and AQP4 in the ventricle region of rats with and without hydrocephalus. Compared with the sham group, AQP1 in the SAH w/t HyC group was slightly higher at days 1,3 , and 7 , peaking at day 3 (Fig. 4A). Simi-

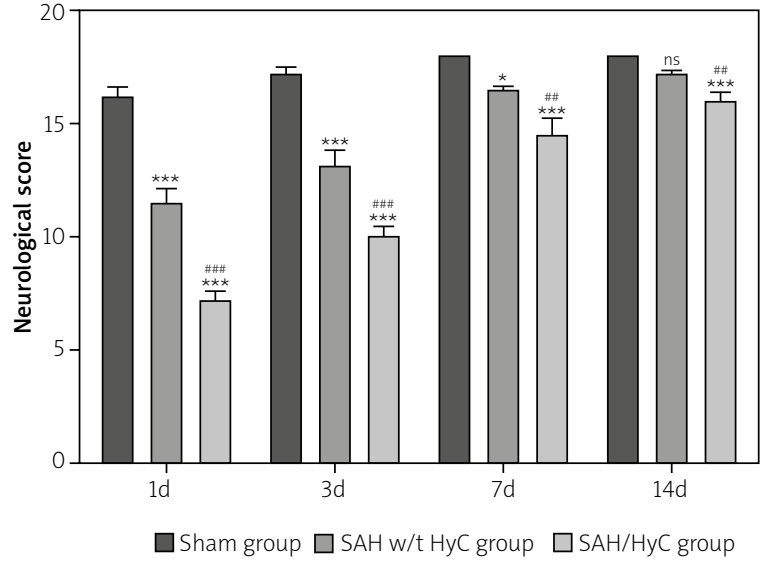

Fig. 2. Evaluation of the neurologic dysfunctions in rats after SAH. SAH/HyC caused a worse neurological function at days 1, 3, 7, and 14. Values are expressed as the mean \pm SEM. Compared with the sham group, ${ }^{* * *} p<0.001,{ }^{* *} p<0.01,{ }^{*} p<0.05$, ns,

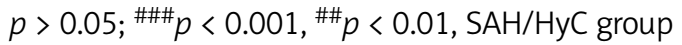
vs. SAH w/t HyC group. For the sham group, $n=6$ at day $1, n=6$ at day $3, n=6$ at day 7 , and $n=6$ at day 14; for the SAH w/t HyC group, $n=13$ at day $1, n=10$ at day $3, n=12$ at day 7 , and $n=13$ at day 14 ; for the SAH/HyC group, $n=7$ at day 1 , $n=11$ at day $3, n=9$ at day 7 , and $n=9$ at day 14 .

larly, AQP4 in the SAH w/t HyC group were slightly higher at days 1 and 3, peaking at day 3 (Fig. 4B). Compared with the sham group, the expression of AQP1 (Fig. 4A) and AQP4 (Fig. 4B and Supplementary Figure 1) in the $\mathrm{SAH} / \mathrm{HyC}$ group was higher at all time points. Compared with the SAH w/t HyC group, AQP1 and AQP4 in the SAH/HyC group also peaked at day 3 . In addition, AQP1 staining could be found in the intracellular region of choroid plexus epithelium at day 1 (Fig. 4A). In hydrocephalic rats, the higher AQP1 was mainly located at both the apical membrane and the basolateral membrane of the choroid plexus epithelium (Fig. 4A). On the contrary, the higher AQP4 was predominantly observed in ependymal cells (Fig. 4B). 
A

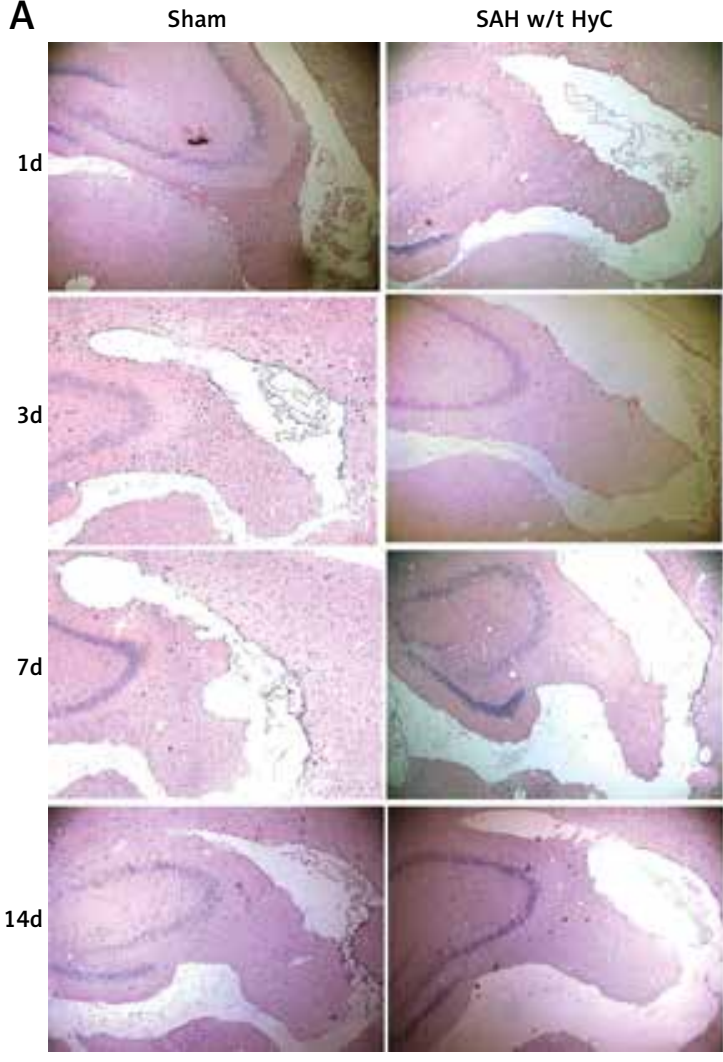

$\mathrm{SAH} / \mathrm{HyC}$

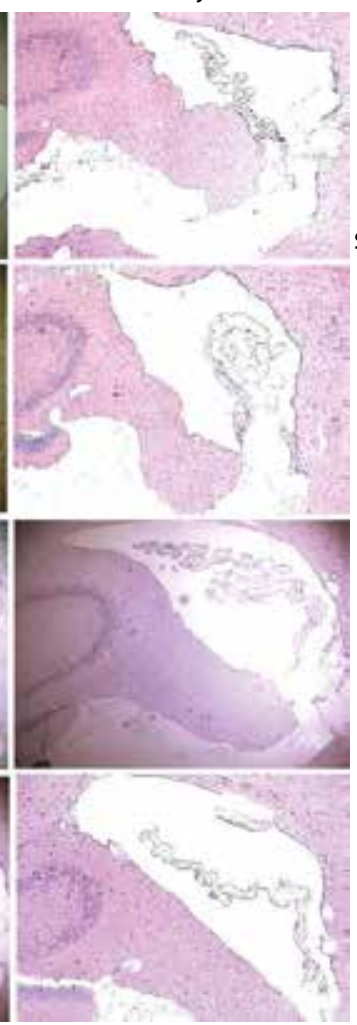

B preoptic area striatum hippocampus

Sham
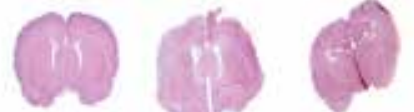

SAH w/t HyC
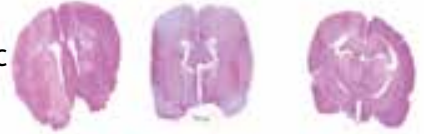

SAH/HyC
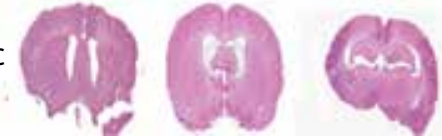

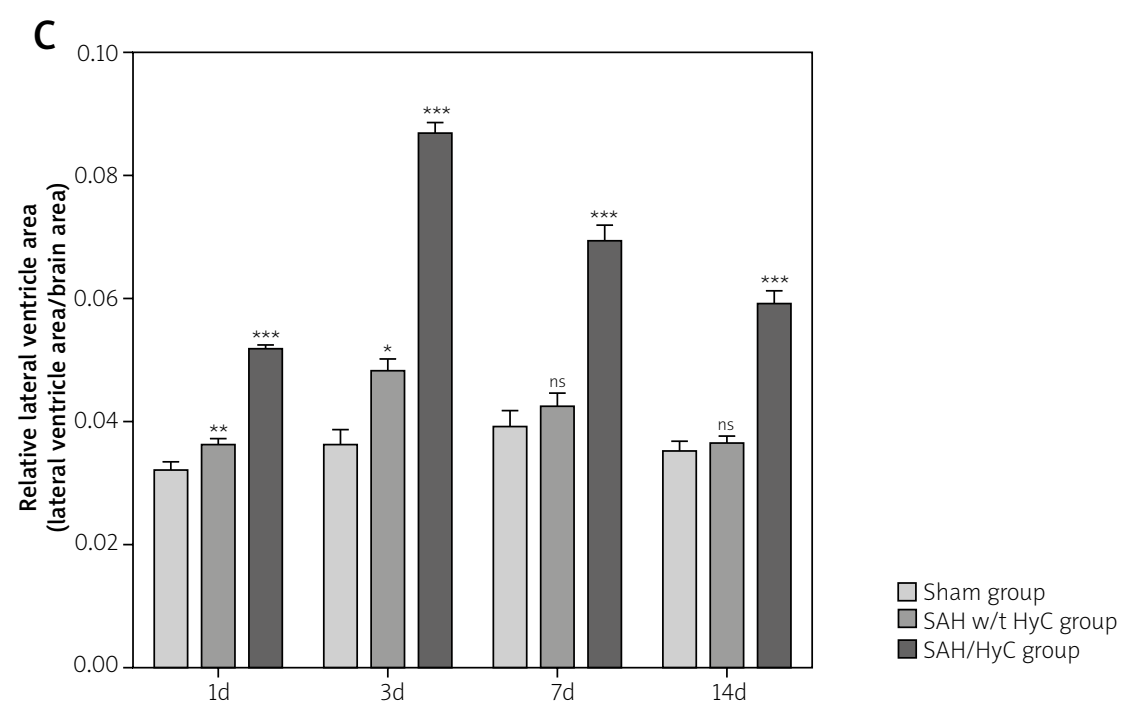

Fig. 3. Assessment of hydrocephalus in rats after SAH by HE staining. A) Sections at the level of the hippocampus for the sham group, the SAH/HyC group and the SAH w/t HyC group at each time point were examined by HE staining. Magnification, 5×. B) Representative photographs showed the HE staining of the sham group, the SAH/HyC group and the SAH w/t HyC group at the level of the preoptic area, the striatum, and the hippocampus at day 3. Scale bar, $3 \mathrm{~mm}$. C) SAH caused a significant increase in relative lateral ventricle areas at all time points, and the increase at day 3 was the most obvious. Values are expressed as the mean \pm SEM. Compared with the sham group, ${ }^{* * *} p<0.001,{ }^{* *} p<0.01,{ }^{*} p<0.05, \mathrm{~ns}, p>0.05$. 

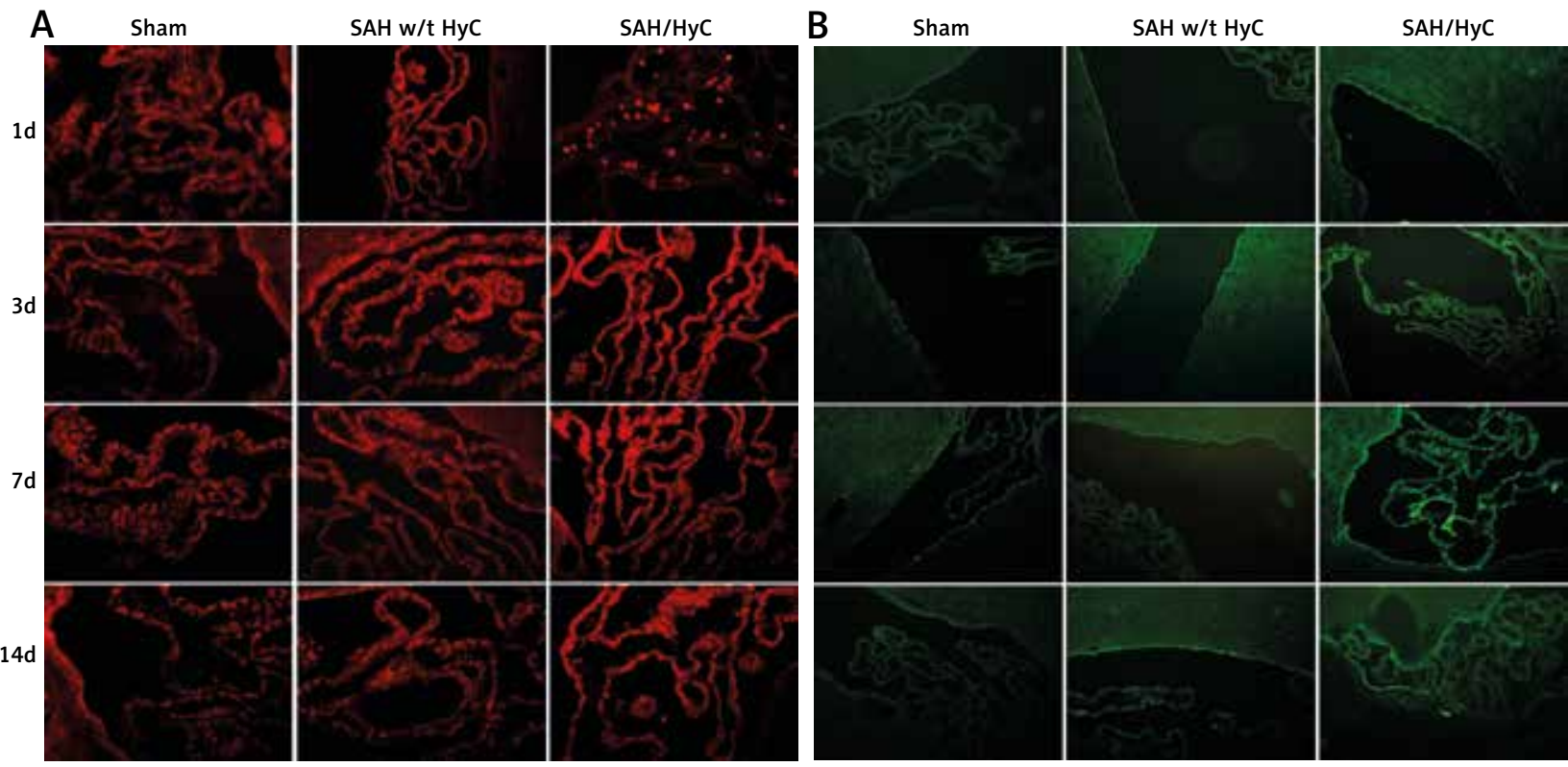

Fig. 4. Expression of $A Q P 1$ and $A Q P 4$ in the ventricle region of rats after SAH. The levels of AQP1 (A) and AQP4 (B) immunofluorescence staining of the ventricle region were evaluated in rats from the sham group, the SAH w/t HyC group, and the SAH/HyC group. Strong staining of AQP1 and AQP4 could be seen in the $\mathrm{SAH} / \mathrm{HyC}$ group at each time point. The staining of AQP1 and AQP4 was most obvious at day 3. AQP1 staining can be observed in the intracellular region of choroid plexus epithelium at day 1 . Scale bar, $50 \mu \mathrm{m}$.

A

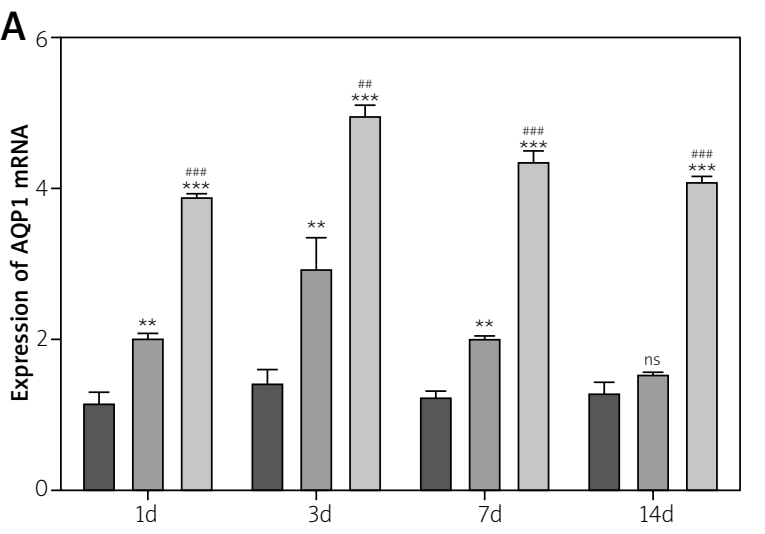

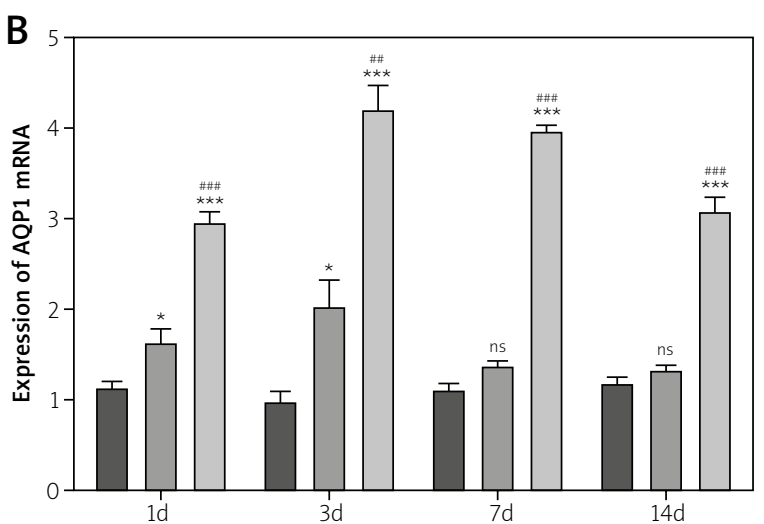

$\square$ Sham group $\square$ SAH w/t HyC group $\square$ SAH/HyC group

Fig. 5. Changes of $A Q P 1$ and $A Q P 4$ mRNA expression in the peripheral region of the lateral ventricle and in the lateral ventricle region after SAH/HyC. The mRNA levels of AQP1 (A) and AQP4 (B) increased significantly at days $1,3,7$, and 14, and peaked at day 3 after $\mathrm{SAH}$-induced hydrocephalus. Values are expressed as the mean \pm SEM. Compared with the sham group, ${ }^{* *} p<0.001,{ }^{* *} p<0.01,{ }^{*} p<0.05$, ns, $p>0.05$; \#\#\# $p<0.001$, \#\# $p<0.01$, the SAH/HyC group vs. the SAH w/t HyC group. For the sham group, $n=3$ at day 1 , $n=3$ at day $3, n=3$ at day 7 , and $n=3$ at day 14 ; for the SAH w/t HyC group, $n=3$ at day $1, n=3$ at day 3 , $n=3$ at day 7 , and $n=3$ at day 14 ; for the SAH/HyC group, $n=3$ at day $1, n=3$ at day $3, n=3$ at day 7 , and $n=3$ at day 14 . 

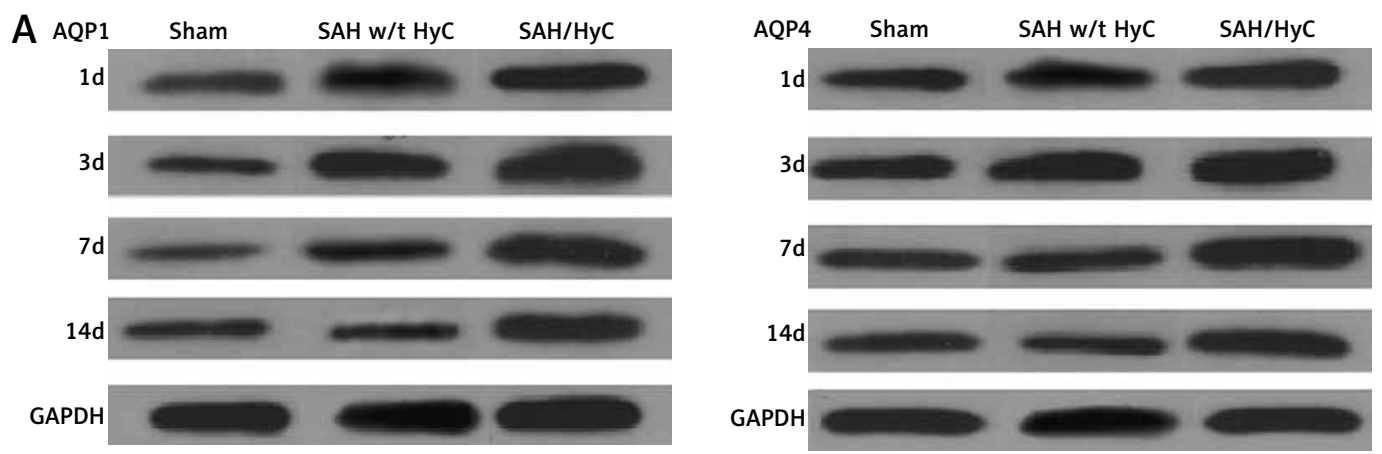

B
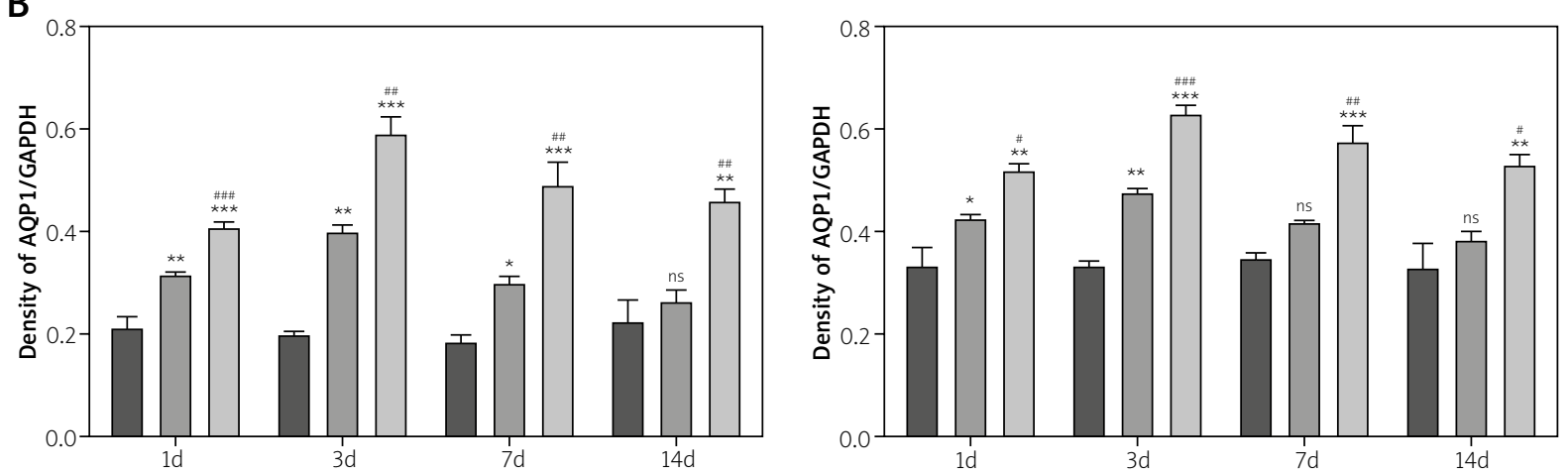

$\square$ Sham group $\square$ SAH w/t HyC group $\square$ SAH/HyC group

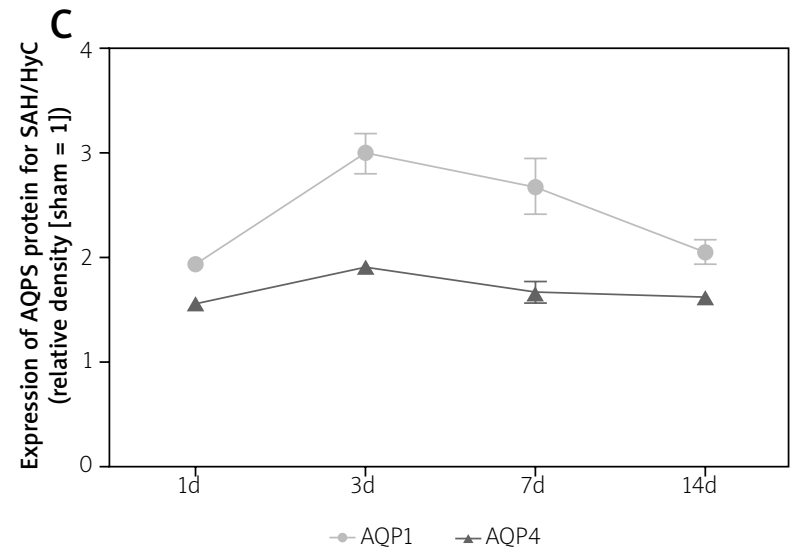

Fig. 6. Changes of AQP1 and AQP4 protein expression in the peripheral region of lateral ventricle and in the lateral ventricle region after SAH/HyC. A) Representative autoradiograms of AQP1 and AQP4 proteins for the sham group, the $\mathrm{SAH} w / \mathrm{t} \mathrm{HyC}$ group and the $\mathrm{SAH} / \mathrm{HyC}$ group at each time point. B) Quantitative analysis of AQP1 and AQP4 protein levels in all groups at indicated time points. Values are expressed as the mean \pm SEM. C) Dynamic changes of relative AQP1 and AQP4 protein expression along with the observation period. Protein levels of AQP1 and AQP4 in the sham group at each time point were arbitrarily defined as 1 .

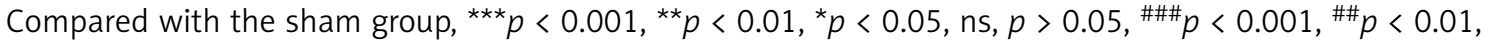
${ }^{\#} p<0.05$, the SAH/HyC group vs. the SAH w/t HyC group. For the sham group, $n=3$ at day $1, n=3$ at day 3 , $n=3$ at day 7 , and $n=3$ at day 14 ; for the SAH w/t HyC group, $n=3$ at day $1, n=3$ at day $3, n=3$ at day 7 , and $n=3$ at day 14; for the SAH/HyC group, $n=3$ at day $1, n=3$ at day $3, n=3$ at day 7 , and $n=3$ at day 14 . 

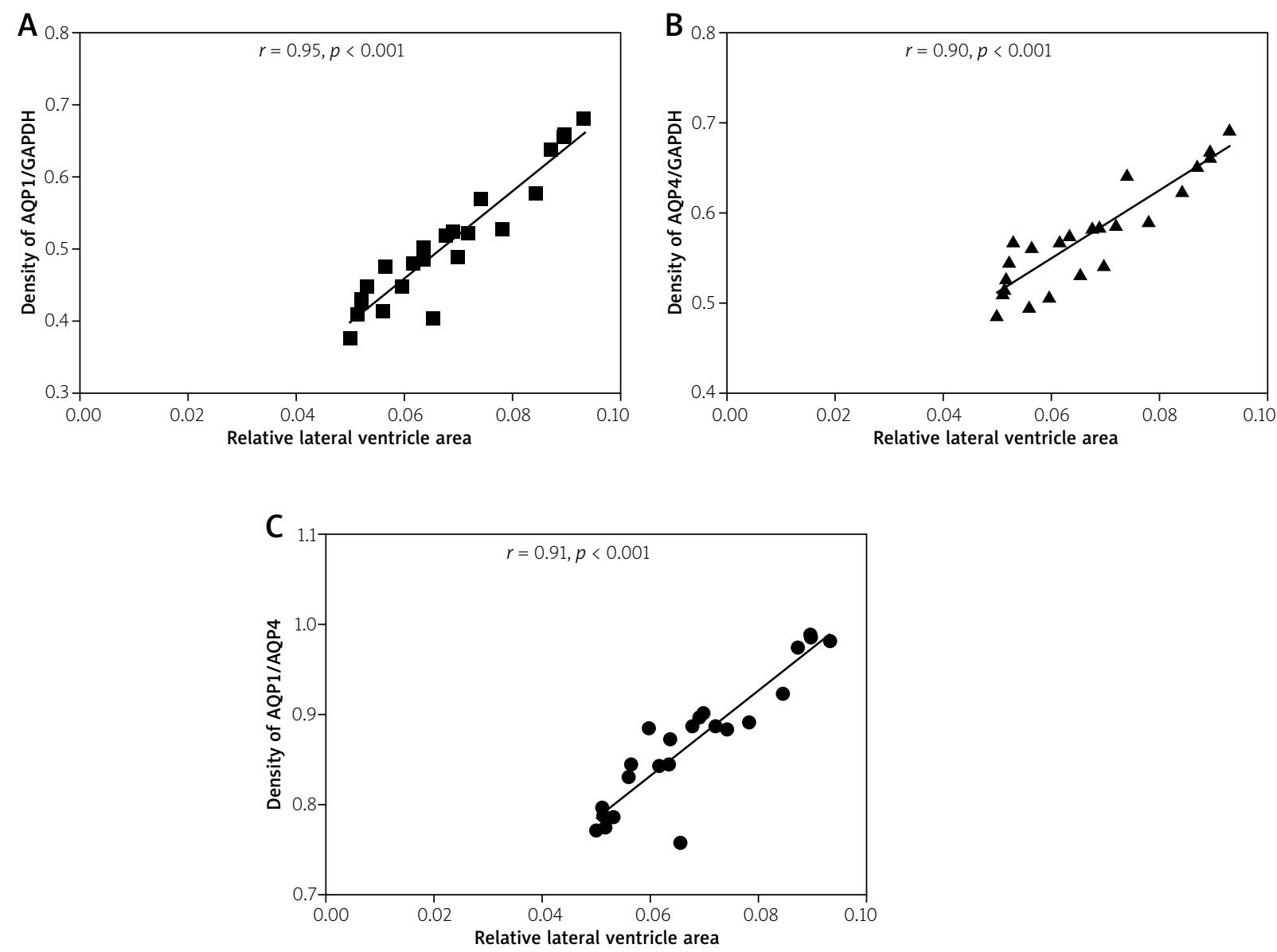

Fig. 7. Correlation between AQP1 or AQP4 protein expression and the relative lateral ventricle area in rats after $\mathrm{SAH} / \mathrm{HyC}$. Plots of the relative lateral ventricle area versus relative protein levels of AQP1 (A), relative protein levels of AQP4 (B), and relative AQP1/AQP4 proteins ratios (C) in the SAH/HyC group are shown. For the sham group, $n=3$ at day $1, n=3$ at day $3, n=3$ at day 7 , and $n=3$ at day 14 ; for the SAH w/t HyC group, $n=3$ at day $1, n=3$ at day $3, n=3$ at day 7 , and $n=3$ at day 14 ; for the SAH/HyC group, $n=3$ at day $1, n=3$ at day $3, n=3$ at day 7 , and $n=3$ at day 14 .

\section{mRNA and protein expression of AQP1 and AQP4 in the ventricle region}

The mRNA expression of AQP1 and AQP4 in the peripheral region of the lateral ventricle was detected by RT-qPCR. Compared with the sham group, AQP1 mRNA in the SAH w/t HyC group was significantly higher at days 1, 3, and 7 (Fig. 5, $p<0.01$ ), and peaked at day 3. AQP4 mRNA in the SAH w/t HyC group was significantly higher at days 1 and 3 (Fig. 5, $p<0.05$ ), and peaked at day 3. In the SAH/ HyC group, the mRNA levels of AQP1 and AQP4 were significantly higher at all time points (Fig. 5, $p<0.001)$. They both peaked at day 3. Compared with the $\mathrm{SAH}$ w/t HyC group, the $\mathrm{SAH} / \mathrm{HyC}$ group had a higher expression of AQP1 and AQP4 mRNA at all time points (Fig. $5, p<0.01$ ).

We also evaluated the expression of AQP1 and AQP4 in the peripheral region of the lateral ventricle by western blot (Fig. 6A). The same trends of AQP1 and AQP4 expressions were observed the protein level (Fig. 6B and Supplementary Figure 2) compared to mRNA levels. Compared with the SAH w/t HyC group, the $\mathrm{SAH} / \mathrm{HyC}$ group had a significantly higher expression of AQP1 and AQP4 protein at all time points (Fig. 6B, $p<0.05$ ). Remarkably, the expres- 
sion curve in time indicated that AQP1 had a more increase than AQP4 (Fig. 6C), probably due to more critical roles of AQP1 in the production of CSF [14]. Taken together, these results suggest that SAH and hydrocephalus significantly affect the expression of AQP1 and AQP4 in the rat lateral ventricle.

\section{Correlation between AQP1/AQP4 expression and relative lateral ventricle area after hydrocephalus induced by SAH}

Linear regression analyses were performed in order to explore the possible relevance between AQP1/AQP4 expression and the relative lateral ventricle area. In the SAH/HyC group, positive correlations were observed between the relative lateral ventricle area and the expression of AQP1 $(r=0.95, p<$ 0.001 , Fig. 7A) and AQP4 ( $r=0.90, p<0.001$, Fig. 7B) proteins. The relative lateral ventricle area was positively correlated to the ratio of $\mathrm{AQP} 1$ and AQP4 protein levels ( $r=0.91, p<0.001$, Fig. 7C) in the SAH/ HyC group. In the SAH w/t HyC group, the lateral ventricle area had no correlation with the ratio of AQP1 and AQP4 protein levels $(r=0.31, p>0.05$, data not shown). This suggested that the expression of AQP1 may be more correlated to the degree of hydrocephalus induced by SAH, and the increase in AQP1 may contribute to the development of hydrocephalus. Indeed, the relative ratio of the $\mathrm{AQP} 1$ and $\mathrm{AQP} 4$ proteins in the $\mathrm{SAH} / \mathrm{HyC}$ group was higher than that in the SAH w/t HyC group $(0.86 \pm 0.01$ vs. $0.74 \pm 0.01$, $p<0.001)$. The ratio of AQP1 and AQP4 expression in the SAH w/t HyC group could be expressed as $0.742 \pm 0.081$ (mean \pm SDs). According to the $95 \%$ upper limit of reference value for the ratio of AQP1 and AQP4 expression in the SAH w/t HyC group, the ratio of 0.864 may be considered as the threshold for hydrocephalus.

\section{Discussion}

The biomolecular mechanisms underlying hydrocephalus after SAH are still not addressed. Although AQP channels are found to play an important role in the brain water homeostasis, their exact roles in the pathophysiology of hydrocephalus still remain obscure. This study showed the dynamic changes of AQP1 and AQP4 in hydrocephalus after SAH by injection of autologous blood into the cisterna magna in rats. We found that the expression of AQP1 and AQP4 increased after hydrocephalus induced by $\mathrm{SAH}$, and peaked at day 3. The expression of AQP1 and AQP4, as well as the ratio of AQP1/AQP4, are positively correlated with the relative lateral ventricle area in rats with hydrocephalus. Our results suggest that AQP1 and AQP4 might be involved in the pathogenesis of SAH-induced hydrocephalus.

According to its occurrence timing, hydrocephalus is classified into three forms: acute (0-3 days after haemorrhage), subacute (4-13 days after haemorrhage), and chronic ( $\geq 14$ days after haemorrhage) [23]. The period of hydrocephalus in the present study included the three time stages from day 1 to day 14 , and we could observe the dynamic change of hydrocephalus severity over time after SAH. Up to now, authors think that different mechanisms underlie the different phases of SAH hydrocephalus. Most of them support the traditional view that non-communicating hydrocephalus usually appears in the acute stage with abnormal cerebrospinal fluid circulation, mainly because of the mass effect or blood clots in the ventricles, while communicating hydrocephalus is usually seen in the chronic stage with reabsorption disorder of CSF due to inflammatory damage and subsequent fibrosis and adhesion of leptomeninges [38].

A study by Kanat et al. showed that haemorrhage in the early phase (2 days) could trigger CSF secretion in aneurysmal SAH, but it is not possible in the late phase (14 days) because of choroid plexus degeneration [32]. Several previous studies indicated that blood clot and blood components such as iron, hemosiderin, and thrombin could disturb CSF circulation throughout the progress of acute and chronic hydrocephalus $[15,16,20]$. Considering the clearance and metabolism of the blood over time, we think that the change of CSF reabsorption and secretion in different stages may be the main cause of dynamic changes of hydrocephalus after SAH in the present study. Some authors consider that blood and a small quantity of thrombin play a main role in early brain injury, and the effect of thrombin and the products of erythrocyte lysis cease after 3 days $[10,27]$. It could explain why hydrocephalus in our experimental model started at day 1 and peaked at day 3. The relative decrease of hydrocephalus at days 7 and 14 may be due to the clearance and metabolism of the blood components.

$A Q P 1$ and AQP4 are the important AQPs in the brain, participating in water balance and some other 
pathophysiological processes. AQP4 was reported to play a role in astrocyte migration, neuroexcitation, neuroinflammation and regulating glutamate transporter-1 (GLT-1) function, while AQP1 is related to pain sensation $[4,21,30]$. Researches demonstrated that AQP1 and AQP4 increase after SAH, which could be related to early brain injury and brain oedema $[3,11,39]$. Skjolding et al. showed that AQP4 expression had a dynamic variation in hydrocephalus induced by kaolin injection. AQP4 decreased in the cortex and periventricular region at day 2 , normalized after 1 week, and increased in periventricular region after 2 weeks [40]. Owler et al. studied the expression and localization of AQP1 in the choroid plexus of mice after hydrocephalus using a cisternal kaolin injection model [36]. AQP1 mRNA had a low level at day 3, but remained unchanged at day 5 . Immunohistochemistry and immunogold electron microscopy showed that AQP1 was located in the cytoplasm of kaolin-injected mice [36]. Similarly, the AQP1 staining in the intracellular region of the choroid plexus epithelium was observed at day 1 in our study. We inferred that this may play a compensatory role in the early stage of hydrocephalus. In addition, in this study, we could see that the increases in AQP1 and AQP4 expression changed over time after $\mathrm{SAH}$, while the increase was more significant in the $\mathrm{SAH} / \mathrm{HyC}$ group than in the $\mathrm{SAH}$ w/t HyC group. We speculated that the increase in the SAH w/t HyC group may associated with early brain injury [40]. The high levels of AQP1 and AQP4 in the SAH/HyC group were positively correlated to the relative lateral ventricle area, which indicated that AQP1 and AQP4 played an important role in the occurrence and development of hydrocephalus induced by SAH.

AQP4 is mainly distributed in the astrocyte end feet around microvessels, glia limitans, subependymal astroglia, and ependyma [4,14], while AQP4 could be on microvessels in the cerebral cortex [24]. AQP1 is chiefly expressed on apical membrane of choroid plexus epithelium, while some reports suggested that AQP1 had a low expression on microvascular endothelial cells and neuron, and on astrocytes after cerebral injury $[4,26,50]$. In the present study, the increased AQP1 was distributed on the apical membrane and the basolateral membrane of choroid plexus epithelium, and increased AQP4 was mainly distributed in ependymal cells in the ventricular region. We could sometimes find a relative high expression of AQP1 on subependymal microvessels and AQP4 on astrocytes in the rat from the SAH/HyC group. The location of AQP1 and AQP4 in this study is supported by previous studies to a certain extent.

Including the present study, studies on different hydrocephalus models showed up-regulation of AQP4 expression. Bloch et al. reported a significant ventriculomegaly and increased ICP and brain water content by $2-3 \%$ in AQP4-knockout mice, compared with the wild-type mice, following a kaolin injection model in cisterna magna [5]. Tourdias et al. studied the communicating inflammatory hydrocephalus induced by lysophosphatidylcholine (LPS), and found that the increase in AQP4 was correlated to CSF volume and periventricular apparent diffusion coefficient (ADC) [43]. AQP4 was found distributed on the whole membrane of hypertrophic astrocytes in the hydrocephalic brain [43]. Studies on different hydrocephalus models showed up-regulation of AQP4 expression. Increased ICP secondary to hydrocephalus driving flow from the ventricles into parenchyma could lead to hydrocephalic brain oedema, in which the increase in AQP4 may be in compensatory regulation of CSF reabsorption across the ependyma $[14,36]$.

The role of AQP1 in hydrocephalus is controversial. Researches pointed out that AQP1 knockout could lead to a 5 -fold reduction in water permeability of choroid epithelium cells, 20-25\% reduction in the CSF production, and $56 \%$ reduction in ICP when compared with wild type mice [14,35]. Mao et al. reported that $A Q P 1$ had no change in kaolin-induced hydrocephalus [31]. Smith et al. found that AQP1 decreased in a case of choroid plexus hyperplasia induced by non-obstructive hydrocephalus using immunohistochemistry, and proposed a compensatory role of AQP1 in hydrocephalus [41]. But in a case of communicative hydrocephalus induced by choroid plexus papilloma, the increase in AQP1 expression was reported [29].

In this study, the dramatic change of AQP1 and AQP4 expression, and the positive correlation between the relative lateral ventricle area and the expression of AQP1 and AQP4 protein or the ratio of AQP1 and AQP4 protein expression, could support the above points of view. AQP1 has an important role in the production of CSF, and AQP4 takes part in hydrocephalus compensation. In addition, through the analysis of the $95 \%$ upper limit of reference value for the ratio of AQP1 and AQP4 in the SAH w/t $\mathrm{HyC}$ group, our results indicated that $\mathrm{SAH}$ could lead 
to the increase in AQP1 protein, and hydrocephalus may occur when the role of AQP1 in promoting hydrocephalus is more dominant than the role of AQP4 in hydrocephalus compensation, which is more likely when the ratio of AQP1/AQP4 is $\geq 0.864$. We infer that blood components could trigger the increase in AQP1 and lead to the production of CSF and hydrocephalus. Furthermore, Castaneyra-Ruiz et al. reported that AQP4 was increased in CSF in cases of communicating hydrocephalus due to ependyma denudation [7]. We consider ependyma degeneration, and denudation might contribute to the relative decrease in AQP4 in the late phase in this study.

\section{Conclusions}

Taken together, our study suggests that SAH could increase the levels of AQP1 and AQP4, whose expressions are remarkably correlated with the severity of hydrocephalus. This underscores the critical roles of AQP1 and AQP4 as important regulation molecules in $\mathrm{SAH}$-induced hydrocephalus, and further implies AQP1 and AQP4 as potential drug targets for developing therapeutic strategies against hydrocephalus.

\section{Funding}

The study was supported by the Hubei Province Department of Health and Family Planning commission (WJ2015MB264) and Yichang Board of Science and Technology (A1530108).

\section{Disclosure}

The authors report no conflict of interest.

Supplementary Figures 1 and 2 are available at the journal's website.

\section{References}

1. Adams H, Ban VS, Leinonen V, Aoun SG, Huttunen J, Saavalainen T, Lindgren A, Frosen J, Fraunberg M, Koivisto T, Hernesniemi J, Welch BG, Jaaskelainen JE, Huttunen TJ. Risk of shunting after aneurysmal subarachnoid hemorrhage: a collaborative study and initiation of a consortium. Stroke 2016; 47: 2488-2496.

2. Agre P. Nobel lecture. Aquaporin water channels. Biosci Rep 2004; 24: 127-163.

3. Badaut J, Brunet JF, Grollimund L, Hamou MF, Magistretti PJ, Villemure JG, Regli L. Aquaporin 1 and aquaporin 4 expression in human brain after subarachnoid hemorrhage and in peritumoral tissue. Acta Neurochir Suppl 2003; 86: 495-498.
4. Badaut J, Fukuda AM, Jullienne A, Petry KG. Aquaporin and brain diseases. Biochim Biophys Acta 2014; 1840: 1554-1565.

5. Bloch O, Auguste KI, Manley GT, Verkman AS. Accelerated progression of kaolin-induced hydrocephalus in aquaporin-4-deficient mice. J Cereb Blood Flow Metab 2006; 26: 1527-1537.

6. Carbrey JM, Agre P. Discovery of the aquaporins and development of the field. Handb Exp Pharmacol 2009; 190: 3-28.

7. Castaneyra-Ruiz L, Gonzalez-Marrero I, Gonzalez-Toledo JM, Castaneyra-Ruiz A, de Paz-Carmona H, Castaneyra-Perdomo A, Carmona-Calero EM. Aquaporin-4 expression in the cerebrospinal fluid in congenital human hydrocephalus. Fluids Barriers CNS 2013; 10: 18.

8. Chen D, Tang J, Khatibi NH, Zhu M, Li Y, Wang C, Jiang R, Tu L, Wang S. Treatment with Z-ligustilide, a component of Angelica sinensis, reduces brain injury after a subarachnoid hemorrhage in rats. J Pharmacol Exp Ther 2011; 337: 663-672.

9. Chen S, Luo J, Reis C, Manaenko A, Zhang J. Hydrocephalus after subarachnoid hemorrhage: pathophysiology, diagnosis, and treatment. Biomed Res Int 2017; 2017: 8584753.

10. Chen Z, Gao C, Hua Y, Keep RF, Muraszko K, Xi G. Role of iron in brain injury after intraventricular hemorrhage. Stroke 2011; 42: 465-470.

11. Chu H, Huang C, Ding H, Dong J, Gao Z, Yang X, Tang Y, Dong Q. Aquaporin-4 and cerebrovascular diseases. Int J Mol Sci 2016; 17: pii: E1249.

12. de Oliveira JG, Beck J, Setzer M, Gerlach R, Vatter H, Seifert V, Raabe A. Risk of shunt-dependent hydrocephalus after occlusion of ruptured intracranial aneurysms by surgical clipping or endovascular coiling: a single-institution series and meta-analysis. Neurosurgery 2007; 61: 924-933; discussion 933-924.

13. Dudhani RV, Kyle M, Dedeo C, Riordan M, Deshaies EM. A low mortality rat model to assess delayed cerebral vasospasm after experimental subarachnoid hemorrhage. J Vis Exp 2013; 71: e4157.

14. Filippidis AS, Kalani MY, Rekate HL. Hydrocephalus and aquaporins: lessons learned from the bench. Childs Nerv Syst 2011; 27: 27-33.

15. Gao C, Du H, Hua Y, Keep RF, Strahle J, Xi G. Role of red blood cell lysis and iron in hydrocephalus after intraventricular hemorrhage. J Cereb Blood Flow Metab 2014; 34: 1070-1075.

16. Gao F, Liu F, Chen Z, Hua Y, Keep RF, Xi G. Hydrocephalus after intraventricular hemorrhage: the role of thrombin. J Cereb Blood Flow Metab 2014; 34: 489-494.

17. Germanwala AV, Huang J, Tamargo RJ. Hydrocephalus after aneurysmal subarachnoid hemorrhage. Neurosurg Clin N Am 2010; 21: 263-270.

18. Hatefi M, Azhary S, Naebaghaee H, Mohamadi HR, Jaafarpour $M$. The effect of fenestration of lamina terminalis on the vasospasm and shunt-dependent hydrocephalus in patients following subarachnoid haemorrhage. J Clin Diagn Res 2015; 9: Pc15-18.

19. Hu Q, Vakhmjanin A, Li B, Tang J, Zhang JH. Hyperbaric oxygen therapy fails to reduce hydrocephalus formation following subarachnoid hemorrhage in rats. Med Gas Res 2014; 4: 12.

20. Hua Y, Keep RF, Hoff JT, Xi G. Brain injury after intracerebral hemorrhage: the role of thrombin and iron. Stroke 2007; 38: 759-762. 
21. Huber VJ, Tsujita M, Nakada T. Aquaporins in drug discovery and pharmacotherapy. Mol Aspects Med 2012; 33: 691-703.

22. Kilic M, Yilmaz I, Tanriverdi O, Akgun C, Musluman AM, Yilmaz A. Factors that affect postoperative hydrocephalus development in aneurysmal subarachnoid hemorrhage: a clinical study. Turk Neurosurg 2017; 27: 353-361.

23. Kim JM, Jeon JY, Kim JH, Cheong JH, Bak KH, Kim CH, Yi HJ, Kim KM. Influence of lamina terminalis fenestration on the occurrence of the shunt-dependent hydrocephalus in anterior communicating artery aneurysmal subarachnoid hemorrhage. J Korean Med Sci 2006; 21: 113-118.

24. Kobayashi H, Yanagita T, Yokoo H, Wada A. Molecular mechanisms and drug development in aquaporin water channel diseases: aquaporins in the brain. J Pharmacol Sci 2004; 96: 264-270.

25. Lackner P, Vahmjanin A, Hu Q, Krafft PR, Rolland W, Zhang JH. Chronic hydrocephalus after experimental subarachnoid hemorrhage. PLoS One 2013; 8: e69571.

26. Lee JH, Cui HS, Shin SK, Kim JM, Kim SY, Lee JE, Koo BN. Effect of propofol post-treatment on blood-brain barrier integrity and cerebral edema after transient cerebral ischemia in rats. Neurochem Res 2013; 38: 2276-2286.

27. Lee JY, Keep RF, He Y, Sagher O, Hua Y, Xi G. Hemoglobin and iron handling in brain after subarachnoid hemorrhage and the effect of deferoxamine on early brain injury. J Cereb Blood Flow Metab 2010; 30: 1793-1803.

28. Livak KJ, Schmittgen TD. Analysis of relative gene expression data using real-time quantitative PCR and the 2(-Delta Delta $C(T))$ Method. Methods 2001; 25: 402-408.

29. Longatti P, Basaldella L, Orvieto E, Dei Tos A, Martinuzzi A. Aquaporin(s) expression in choroid plexus tumours. Pediatr Neurosurg 2006; 42: 228-233.

30. Mader S, Brimberg L. Aquaporin-4 Water Channel in the Brain and Its Implication for Health and Disease. Cells 2019; 8: pii E90.

31. Mao X, Enno TL, Del Bigio MR. Aquaporin 4 changes in rat brain with severe hydrocephalus. Eur J Neurosci 2006; 23: 2929-2936.

32. Niemela M, Marbacher S. Acute hydrocephalus after subarachnoid hemorrhage - can it be caused by water vesicles of choroid plexuses? World Neurosurg 2013; 80: 307-308.

33. Okubo S, Strahle J, Keep RF, Hua Y, Xi G. Subarachnoid hemorrhage-induced hydrocephalus in rats. Stroke 2013; 44: 547-550.

34. Oliva AA, Jr., Kang Y, Truettner JS, Sanchez-Molano J, Furones C, Yool AJ, Atkins CM. Fluid-percussion brain injury induces changes in aquaporin channel expression. Neuroscience 2011; 180: 272-279.

35. Oshio K, Watanabe H, Song Y, Verkman AS, Manley GT. Reduced cerebrospinal fluid production and intracranial pressure in mice lacking choroid plexus water channel Aquaporin-1. Faseb J 2005; 19: 76-78.

36. Owler BK, Pitham T, Wang D. Aquaporins: relevance to cerebrospinal fluid physiology and therapeutic potential in hydrocephalus. Cerebrospinal Fluid Res 2010; 7: 15.

37. Papadopoulos MC, Verkman AS. Aquaporin water channels in the nervous system. Nat Rev Neurosci 2013; 14: 265-277.
38. Shah AH, Komotar RJ. Pathophysiology of acute hydrocephalus after subarachnoid hemorrhage. World Neurosurg 2013; 80: 304-306.

39. Shi SS, Zhang HB, Wang CH, Yang WZ, Liang RS, Chen Y, Tu XK. Propofol attenuates early brain injury after subarachnoid hemorrhage in rats. J Mol Neurosci 2015; 57: 538-545.

40. Skjolding AD, Rowland IJ, Sogaard LV, Praetorius J, Penkowa M, Juhler M. Hydrocephalus induces dynamic spatiotemporal regulation of aquaporin-4 expression in the rat brain. Cerebrospinal Fluid Res 2010; 7: 20.

41. Smith ZA, Moftakhar P, Malkasian D, Xiong Z, Vinters HV, LaZareff JA. Choroid plexus hyperplasia: surgical treatment and immunohistochemical results. Case report. J Neurosurg 2007; 107: 255-262.

42. Sugawara T, Ayer R, Jadhav V, Zhang JH. A new grading system evaluating bleeding scale in filament perforation subarachnoid hemorrhage rat model. J Neurosci Methods 2008; 167: 327-334.

43. Tourdias T, Dragonu I, Fushimi Y, Deloire MS, Boiziau C, Brochet B, Moonen C, Petry KG, Dousset V. Aquaporin 4 correlates with apparent diffusion coefficient and hydrocephalus severity in the rat brain: a combined MRI-histological study. Neuroimage 2009; 47: 659-666.

44. Vella J, Zammit C, Di Giovanni G, Muscat R, Valentino M. The central role of aquaporins in the pathophysiology of ischemic stroke. Front Cell Neurosci 2015; 9: 108.

45. Verkman AS, Tradtrantip L, Smith AJ, Yao X. Aquaporin water channels and hydrocephalus. Pediatr Neurosurg 2017; 52: 409416.

46. Walcott BP, Iorgulescu JB, Stapleton CJ, Kamel H. Incidence, timing, and predictors of delayed shunting for hydrocephalus after aneurysmal subarachnoid hemorrhage. Neurocrit Care 2015; 23: $54-58$

47. Wang D, Nykanen M, Yang N, Winlaw D, North K, Verkman AS, Owler BK. Altered cellular localization of aquaporin-1 in experimental hydrocephalus in mice and reduced ventriculomegaly in aquaporin-1 deficiency. Mol Cell Neurosci 2011; 46: 318-324.

48. Xie Z, Hu X, Zan X, Lin S, Li H, You C. Predictors of shunt-dependent hydrocephalus after aneurysmal subarachnoid hemorrhage? A systematic review and meta-analysis. World Neurosurg 2017; 106: 844-860.e846.

49. Yool AJ. Aquaporins: multiple roles in the central nervous system. Neuroscientist 2007; 13: 470-485.

50. Zelenina M. Regulation of brain aquaporins. Neurochem Int 2010; 57: 468-488.

51. Zhong Z, Wang B, Dai M, Sun Y, Sun Q, Yang G, Bian L. Carvacrol alleviates cerebral edema by modulating AQP4 expression after intracerebral hemorrhage in mice. Neurosci Lett 2013; 555: 24-29. 